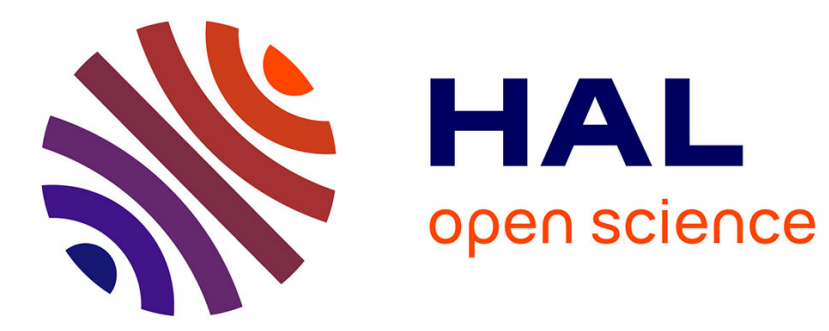

\title{
IBTrack: An ICMP Black holes Tracker
}

Ludovic Jacquin, Vincent Roca, Mohamed Ali Kaafar, Fabrice Schuler, Jean-Louis Roch

\section{To cite this version:}

Ludovic Jacquin, Vincent Roca, Mohamed Ali Kaafar, Fabrice Schuler, Jean-Louis Roch. IBTrack: An ICMP Black holes Tracker. 2012. hal-00695746

\section{HAL Id: hal-00695746 https://hal.science/hal-00695746}

Submitted on 9 May 2012

HAL is a multi-disciplinary open access archive for the deposit and dissemination of scientific research documents, whether they are published or not. The documents may come from teaching and research institutions in France or abroad, or from public or private research centers.
L'archive ouverte pluridisciplinaire HAL, est destinée au dépôt et à la diffusion de documents scientifiques de niveau recherche, publiés ou non, émanant des établissements d'enseignement et de recherche français ou étrangers, des laboratoires publics ou privés. 


\title{
IBTrack: An ICMP Black holes Tracker
}

\author{
Ludovic Jacquin, Vincent Roca, Mohamed Ali Kaafar, Fabrice Schuler \\ Inria, France \\ \{ludovic.jacquin, vincent.roca, mohamed-ali.kaafar, fabrice.schuler\}@inria.fr
}

\author{
Jean-Louis Roch \\ LIG, France \\ jean-louis.roch@imag.fr
}

\begin{abstract}
ICMP is a fundamental part of the Internet as it handles the control and error messages. ICMP's treatment by the network and in particular by different routers it may cross is therefore a key aspect driving troubleshooting and diagnosis processes. In this paper we present IBTrack, a tool that aims at characterizing how the network actually treats different ICMP messages from an user point of view. Specifically, we detail a classification algorithm to categorize router behaviors and we introduce its associated refining method which exploits multiple probing protocols. We illustrate the average Internet router behavior and path composition through results gathered from Planet-Lab nodes using a large CAIDA's snapshot of routed /24. We further show that our refining method improves the routers behavior characterization up to $10 \%$ for more than $1 \%$ of the total number of observed routers.
\end{abstract}

\section{INTRODUCTION}

The Internet Control Message Protocol (ICMP) is one of the main protocols used on Internet and more generally on IPV 4 and IPV 6 networks. It is responsible for transmitting control and error messages over the network (such as routing control, packet treatment error, etc). Although it operates at the second level of the OSI stacks, it is encapsulated on an IP packet.

An example of the ICMP importance is its key implication in the Path Maximun Transmission Unit Discovery (PMTUD) mechanism [1], [2], [3], which has been developed to avoid IP fragmentation. For instance in IPv4, it sets the don't fragment bit (which is not needed in IPV 6 since the protocol does not support router fragmentation). If a router cannot transmit the packet because of its size, it must send back to the source an ICMP "Too Big" (type 3 code 4 on IPv4 and type 2 code 0 on IPv6) packet. Iteratively, the source will lower the size of the packet to match the lowest MTU on a path. The importance of a well-chosen PMTU has been discussed in several previous works, and one key aspect to consider from routers perspective is the number of packets per second to handle[4], [5]. In essence, using the highest possible PMTU value results in a significant bandwidth improvement [6] since the packets treatment overhead remains the same regardless of the packet's size. ICMP routing issues can therefore lead to serious connection problems because of their tight links with the PMTU discovery protocol for example.

This motivates our study that aims at analyzing issues related to ICMP packet processing by routers, and in particular so-called "ICMP black holes". The tool presented in this paper, IBTrack, provides users with a thorough analysis of the routers behavior that lie along the path from a source host controlled by the user and any given destination (that is not assumed to be under control). In particular, IBTrack characterizes the routers forging of ICMP error packets and their transport the way back to the source host. It is also important to note that IBTrack should be a "lightweight" tool which only relies on measures performed by the user at the sending host, without requiring any additional resource (i.e. there is no external monitoring and/or vantage point nor any collaboration from the destinations). We further require that IBTrack does not involve any long-term measurement, so it could be used in a timely basis, when a user needs to establish a diagnosis and locate any possible problem origin (similarly to the ping tool).

Let us first compare IBTrack to some related works. In [7], authors describe Reverse traceroute a tool that can be used to perform measurement (using ICMP) at the level of Internet routers back to a specified host. The tool is capable of examining the router-level topology and does not look into the behaviors of routers to forge different types of ICMP packets when needed. Authors of Reverse Traceroute also assume users do have access to multiple vantage points distributed across the Internet. Our approach is similar to the concept explored in [8], where authors propose a system called Hubble which detects IP-level black holes at a global level of IP connectivity. Besides the fact that Hubble needs periodic probing campaigns of the Internet, which IBTrack tries to avoid, our proposed tool aims to further characterize the reasons to which any ICMP -connectivity related issues are due and provides a fine-grained analysis.

The rest of this paper is organized as follows. Section II presents the model used to describe routers and the assumptions made in our work. section III describes the IBTrack algorithms. Section IV focuses on the methodology and tools used for our measurements. Next, section V presents the results from both coarse-grained Internet and fine-grained ISP levels. Finally, section VI concludes the paper.

\section{ASSUMPTIONS AND TAXONOMY}

In this section, we define the terminology used to define the source and destination path components. We also take a closer look at the restrictions implied by IBTrack's main goals and our practical assumptions. 


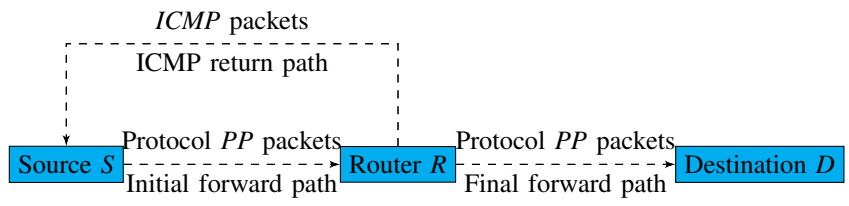

Fig. 1: Paths definitions.

\section{A. Path definitions}

Let us consider a path from a source $S$ to a destination $D$, and a router $R$ along this path (there are typically several routers and $R$ is one of them). We assume that the packet flow generated by $S$ and destined to $D$ uses the probing protocol $P P$. More specifically in this work we consider either ICMP/IP, UDP/IP, or TCP/IP, where IP denotes either IPv4 or IPv6. Figure 1 illustrates the following definitions:

- the initial forward path is the path between $S$ and $R$;

- the final forward path is the path between $R$ and $D$;

- and the ICMP return path is the path taken by ICMP packets destined to $S$ and either generated by $R$ or by a router on the final forward path and that go through $R$;

It is important to note that the initial forward paths and the return ICMP paths are not necessarily identical: routers often use different forwarding strategies depending of routing policies. The forward initial or final paths also potentially depend on the nature of $P P$, since routers often use protocoldependant forwarding rules. Finally, there are potentially as many ICMP return paths as the number of routers on the forwarding paths.

\section{B. Assumptions}

The algorithms we introduce only take into account the information gathered by the source. The main goal of our approach is to characterize routers behavior along a path in order to help users when troubleshooting connectivity issues that are potentially caused by ICMP packets. Therefore we deliberately chose not to rely on external data from vantage point or from the destination during the analysis.

We assume that the routing only depends on the packet's protocol and destination. This is a strong assumption that is however reasonnable in current Internet.

Furthermore, the property used to characterize a router is considered as global, independently from the router's IP interface that has been used during the measurement inferring this property. In other words, we assume that all IP interfaces of that router behave identically.

Finally, since we assume we only control the source $S$, it is worth noticing that the only ICMP return packet type we can trigger from any router on the network is the Time Exceeded type, which is in theory forged when a packet reaches a router with the IP field "Time To Live" equal to zero). During our measurements, will examine ICMP return paths only by using ICMP TTL packets.

\section{Router properties taxonomy}

Let us now consider a certain probing protocol $P P$ used by the source. For this protocol $P P$, each router along the given path is characterized by the following three key properties:

1) Property P1: " $R$ forwards all packets of type $P P$ towards $D$ ".

Said differently, at $R$, each incoming packet on the initial forward path is forwarded on the final forward path. This property, of course, does not imply that these packets reach destination $D$.

2) Property P2: " $R$ is cooperative for packets of type $P P$ ". In case $R$ should send an ICMP packet back to the source, either because of an error (e.g. a packet that exceeds the forwarding link MTU) or because the packet asks for a reply (e.g. in case of an ICMP Echo Request), then the ICMP packet is correctly initialized and sent by $R$ on the ICMP return path.

3) Property P3: "ICMP packets are not filtered by $R$ " and by any router on the ICMP return path from $R$.

When $R$ emits an ICMP packet on the return path, this ICMP packet is routed all the way back to $S$. This property implies that this ICMP packet arrive to $S$, i.e. they are not filtered by any router on the ICMP return path from $R$, which is a strong property. In the particular case where the return path contains one or several routers that are also part of the forward path, this property on $\mathrm{R}$ implies the same property on these routers.

The router behavior can now be expressed as three logical expressions, one for each property. For instance, for a packet type $P: \mathrm{P} 1$. (P2 . ! $3+! \mathrm{P} 2)$ means that router $R$ forwards these packets and is either cooperative (i.e. sends ICMP packets to $S$ ) but these ICMP packets are filtered on the ICMP return path, or is non cooperative (i.e. does not generates ICMP packets back to $S$ ).

\section{THE ICMP BLACK HOLES TRACKING (IBTRACK) TOOL}

In this section we describe our IBTrack tracking algorithm, whose goal is to refine as much as possible the routers behavior, considering only the initial traffic and the backward ICMP path. In a first step, we start by considering a Probing Protocol $P P$ and try to determine the router's behavior. Then, in a second step, we explain how IBTrack can sometimes refine the analysis by crossing the results achieved independently with several probing protocols.

\section{A. IBTrack base algorithm}

The base algorithm is described in figure 2. At the beginning there is no knowledge at all for router $R$, which corresponds to the state at the top of the graph. In that case each property is "undecided" (i.e. it can be true or false), which is expressed by: $P+$ ! .

Then the algorithm is composed of two main steps:

- test the forwarding path through $R$;

- test the ICMP return path from $R$;

We now detail these two steps: 


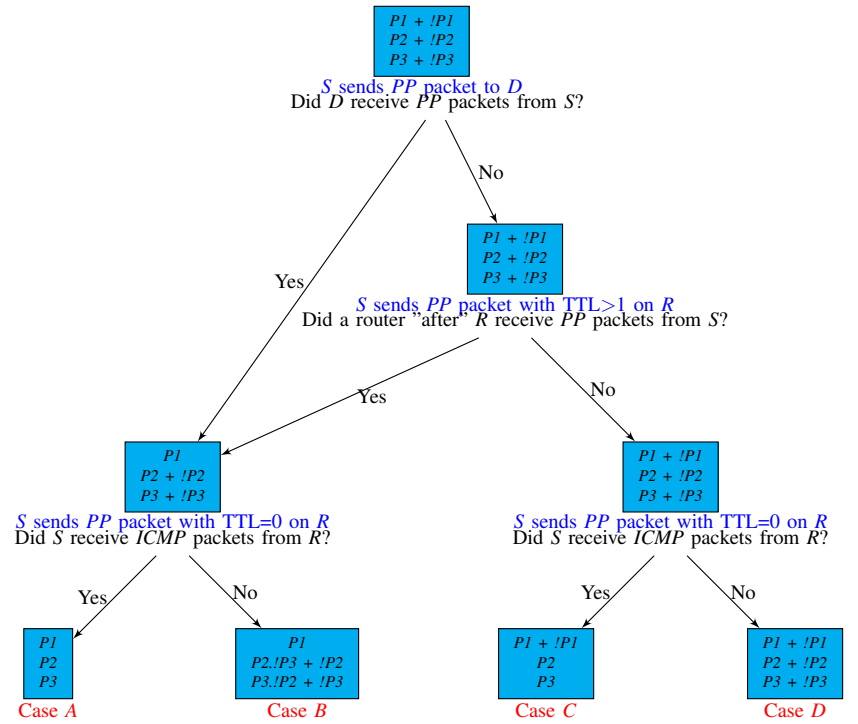

Fig. 2: Base algorithm for router's behavior characterization

1) Forwarding path through $R$ : We first want to test the P1 property (forwarding property towards $D$ ). It can be verified in two ways: either the destination replied, which implies that it has received an incoming packet, or we can assert that a router on the final forward path received an incoming packet. This second method is typical of situations where source $S$ received ICMP packets from a router on the final forward path (i.e. after router $R$ ).

2) ICMP return path from $R$ : We now want to check the P 2 and P 3 properties. For the P 2 property we must verify that the router forges the ICMP error packet and emits it on the ICMP return path. This could be tested by monitoring the ICMP return path. Nevertheless, as we do not control any router of the ICMP return path, we only rely on the reception by the source of the ICMP error packet. For the P 3 property, since it concerns the ICMP return path itself, it can only be evaluated with the reception of ICMP error packets by the source.

3) Classification details: We now present the result of this classification, which consists in four cases, depending on the properties that could be verified or not.

a) Case A: P1.P2.P3

This is the "ideal" router which forwards the initial packets and forges ICMP error packets that all arrive to the source.

b) Case B: P1. (P2.!P $3+! \mathrm{P} 2) \cdot(\mathrm{P} 3 . \mathrm{P} 2+\mathrm{P} 3)$ $\mathrm{P} 1$ is verified, but the interesting part of this case comes from the P 2 property. In this case, the router forwarded the initial packets and also dealt with them (i.e. filtering and packet processing). However the source did not receive any ICMP error packet whereas such a packet should have been forged since the source $S$ sent a packet whose TTL ("Hop Count" in case of $(\mathrm{PV} 6$ ) has been decremented to 0 by $R$ ). It leads to two possibilities:

- $\mathrm{P} 2 \Rightarrow$ !P 3: the ICMP error packet is forged but is blocked along the ICMP return path.

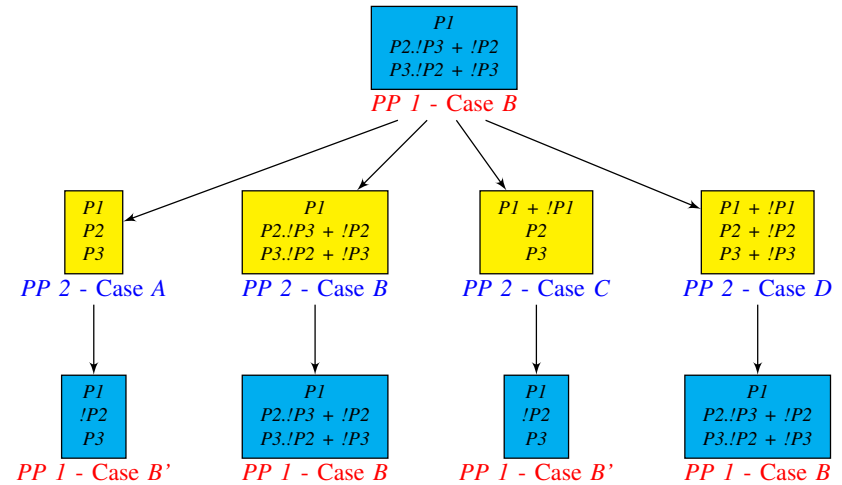

Fig. 3: Refining case $B$ with another probing protocol

- P3 3 !P2: the ICMP return path forwards ICMP packets correctly, however the router $R$ did not not forged any such packet.

c) Case C: (P1+ ! P1).P2.P3

The source gets the ICMP error from the router, but we have no clue that a router on the final forward path received any initial packet.

d) Case D: $(\mathrm{P} 1+! \mathrm{P} 1) \cdot(\mathrm{P} 2+! \mathrm{P} 2) \cdot(\mathrm{P} 3+\mathrm{P} 3)$ For these type of routers, we cannot infer anything because the source did not get back any data.

This base algorithm is applied to every routers in a path (defined by its source and destination) for protocol $P P$, by increasing the TTL value It results in a collection of cases ( $A, B, C$ or $D$ ) for each router along the forward path.

\section{B. IBTrack refining algorithm}

Let us now explain how we can refine the previous algorithm that depends on the probing protocol $P P$ that has been used for probing the network. Let us first notice that the $\mathrm{P} 3$ property concerns exclusively the ICMP return path which is the same independently from the probing protocol $P P^{1}$. Thus, for a given router $R$, we can use the deductions made on the P 3 property using a probing protocol PPl to refine the deductions made using another probing protocol $P P 2$. This is the key idea of our refining algorithm. However, in order to be able to apply this refinement, we must first identify the same router for both probing protocols, whereas we do not necessarily have its identity (it can be a wildcats "*" in a traceroute trace). This aspect is addressed in section IV-C, and for the moment we assume that this assertion as true.

The combination of the results of the algorithm for two distinct probing protocols is detailed in figures 3 and 4 , for router $R$. Since we can only use deductions for the $\mathrm{P} 3$ property, cases $A$ or $C$ cannot be refined (property P 3 is already known in that case). Therefore we focus on cases $B$ and $D$.

\footnotetext{
${ }^{1}$ This is a direct consequence of our assumption that routing only depends on the protocol along the return path, here ICMP, and the destination (here
} $S$ ). 


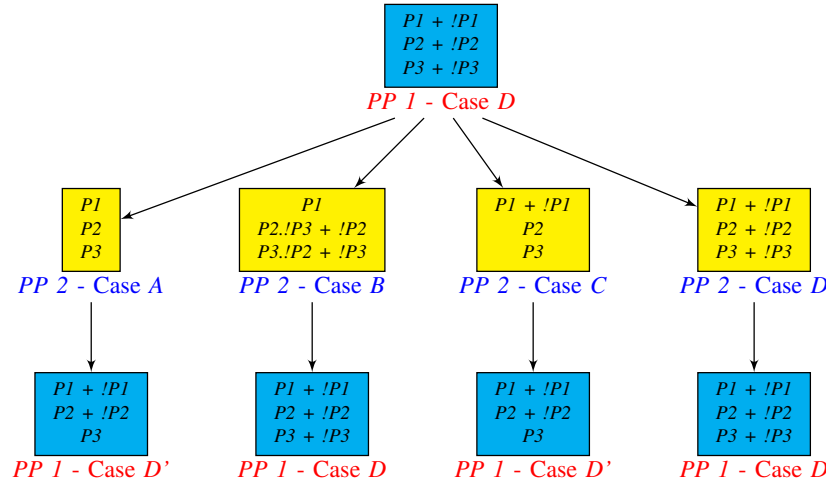

Fig. 4: Refining case $D$ with another probing protocol

\begin{tabular}{|l|l|}
\hline ICMP & UDP \\
\hline 15213.248 .90 .250 & 15213.248 .90 .250 \\
$16 *$ & $16 *$ \\
17205.171 .11 .98 & 17205.171 .11 .98 \\
\hline
\end{tabular}

(a) two $B$ cases

\begin{tabular}{|c|c|}
\hline ICMP & UDP \\
\hline 6195.83 .166 .161 & 6195.83 .166 .161 \\
\hline 7 193.51.181.222 & $7 *$ \\
\hline 8 193.51.189.202 & 8 193.51.189.202 \\
\hline
\end{tabular}

(b) $A$ case and $B$ case

Fig. 5: Two examples of multiple probing protocol results

1) Initial case B for probing protocol PP1 (Fig.3): This is the most interesting case. When property $P 3$ is verified with the second probing protocol, $P P 2$, from the second conditional we can deduce that $\mathrm{P} 3 \Rightarrow$ ! P 2 . Thus, if probing with protocol $P P 2$ ends in cases $A$ or $C$, we can conclude that the $\mathrm{P} 3$ property is verified for protocol $P P 1$, which implies that $\mathrm{P} 2$ is not. This creates a subcase for $B$ that we call $B$ ': $\mathrm{P} 1+$ ! $\mathrm{P} 2+\mathrm{P} 3$.

2) Initial case D for probing protocol PPl (Fig.4): This case can only be refined on the $\mathrm{P} 3$ property itself since there is no material implication or link with another property. This only happens when we end in cases $A$ or $C$ with the second probing protocol.

3) Refining using multiple probing protocols: It is of course possible to use more than one probing protocol to refine the results obtained with $P P 1$. Nevertheless, for this to be true, we must assert that the paths are the same for every combination of the probing protocol and of the refining protocol in order to consider that P 3 property is common.

\section{Examples}

Figure 5 illustrates the refining algorithm through two examples extracted from our experiments.

In the traceroute outputs of figure $5 \mathrm{a}$, the $16^{\text {th }}$ router is a $B$ case for both protocols since: (i) it forwards the initial protocol (the $17^{\text {th }}$ router replied which implies that it has received at least one initial packet); but (ii) no ICMP return packet is received by the source. There is no refining possible in this case and: $\mathrm{P} 1 .(\mathrm{P} 2 . \mathrm{I} 3+! \mathrm{P} 2) \cdot(\mathrm{P} 3 . ! \mathrm{P} 2+\mathrm{P} 3)$

In the traceroutes outputs of figure $5 \mathrm{~b})$, the $7^{\text {th }}$ router is a $B$ case for UDP probing whereas it is an $A$ case for ICMP probing (the ICMP return packet is received by the source). As the ICMP return path is the same (source, protocol and destination are identical), we deduce that for UDP packet the router does not forge the ICMP return packets. The categorization for UDP probing is therefore refined to the $B^{\prime}$ case: $\mathrm{P} 1+! \mathrm{P} 2+\mathrm{P} 3$

The formal model and algorithms being described, the next section will focus on the methodology and on the tools used for Internet measurements.

\section{Measurement methodology}

Before describing the tools and methodology used during the measurements, we detail how we chose the IP addresses to probe.

\section{A. Selection of destination's IP}

To probe a meaningful set of Internet, we used a CAIDA[9] snapshot of the routed / 24 IPV4. This snapshot is composed of a part of the routed / 24 (approximately 10,000) and gives for each a random IP in the $/ 24$. It is important to notice that there is not necessarily a running host behind each IP in the snapshot.

\section{B. Tools}

To perform probing, we used scamper[10] that implements, among others, the paris-traceroute[11] functionnality over the Planet-Lab[12] nodes.

1) Paris-traceroute: This is an improved version of the classical traceroute tool. In particular this tool reveals more routers and links over the path explored and removes some false links inferred by the usual traceroute. This is therefore highly benefic to our needs.

2) Planet-Lab: This is a world wide network aiming to help academic and industrial researchers focusing on new network services. In our test, it provides multiple servers across the world that we use to probe the same destination set of IP addresses. Therefore it helps us improving the coverage of the possible paths to the selected set of IP addresses.

3) Scamper: This is a program that implements most of the classical Internet measurement tools (like ping or paris-traceroute) that can be launched to run in parallel. In particular it is developed to run on Planet-Lab nodes.

One of the features of scamper we use is that after five unidentified routers (i.e. not responding, thus appearing as "*" in the traceroute output) it stops. Figure 6c shows a traceroute terminating like this. This situation can result from different causes, like for instance a single router filtering packets, or multiple routers not replying. Nevertheless, we will deliberately take into account only the first "*" during our tests. 


\begin{tabular}{|l|l||l|l|}
\hline Protocol 1 & Protocol 2 & Protocol 1 & Protocol 2 \\
\hline 1 R1 & 1 R1 & 1 R1 & 1 R1 \\
$2 *$ & $2 \mathrm{R} 2$ & $2 *$ & $2 \mathrm{R} 2$ \\
$3 *$ & $3 \mathrm{R} 3$ & $3 *$ & $3 \mathrm{R} 3$ \\
$4 *$ & $4 \mathrm{R} 4$ & $4 \mathrm{R} 4$ & $4 *$ \\
$5 *$ & $5 \mathrm{R} 5$ & $5 \mathrm{R} 5$ & $5 *$ \\
$6 \mathrm{R} 6$ & $6 \mathrm{R} 6$ & $6 \mathrm{R} 6$ & $6 \mathrm{R} 6$ \\
\hline
\end{tabular}

(a) Two pairs of traceroute outputs considered as the same path

\begin{tabular}{|l|l|}
\hline Protocol 1 & Protocol 2 \\
\hline $1 \mathrm{R} 1$ & $1 \mathrm{R} 1$ \\
$2 \mathrm{R} 21$ & $2 \mathrm{R} 22$ \\
$3 *$ & $3 \mathrm{R} 3$ \\
$4 \mathrm{R} 41$ & $4 \mathrm{R} 42$ \\
$5 *$ & $5 \mathrm{R} 5$ \\
$6 \mathrm{R} 6$ & $6 \mathrm{R} 6$ \\
\hline
\end{tabular}

(b) Two traceroute outputs considered as the same path when allow-

ing two different routers

\begin{tabular}{l|}
\hline ICMP \\
\hline 12198.108 .23 .21 \\
$13 *$ \\
$14 *$ \\
$15 *$ \\
$16 *$ \\
$17 *$ \\
\hline
\end{tabular}

(c) Example of a traceroute finishing with five unidentified routers

Fig. 6: Examples of the path matching algorithm and scamper output

\section{Identifying common path}

One of the main challenges before applying the refining algorithm which uses multiple probing protocols is to be able to match paths that seem to be different. In principle the presence of an unidentified router on a path (i.e. a "*" in the traceroute output) prevents path matching for the final forwarding path. But this situation must be improved. Indeed, if the router has to be identified to allow the application of our refining algorithm, then we would only perform refining between $A$ and $C$ cases, which is of little interesting because these cases already share the same state for the property P 3 .

1) Handling unknow routers: The first and straightforward assumption we make consists in ignoring unknown routers from a path when matching two paths with the same source and destination but with different probing protocols.

Formally, there is no proof for this assumption, but we are convinced that given the maximum number of consecutive unknown routers we allowed ( 5 before traceroute halts) for a protocol, if two paths share the same number of routers and if the identified routers are the same, then the two paths are most likely the same.

For instance, figure 6a exhibits two situations considered as belonging to the same path by the path matching algorithm.

2) Handling tunnels: We also wanted to add to the path matching algorithm the capability to identify tunnels. More particularly, we want to isolate path divergences due to perflow routing (for example through MPLS tunnel[13]).

Therefore, we improved our algorithm by allowing some consecutive identified routers to be different on the same path. This feature adds itself to the previous one; thus "consecutive identified routers" do not involve potential unknown routers. Nevertheless, the number of routers and their position on the

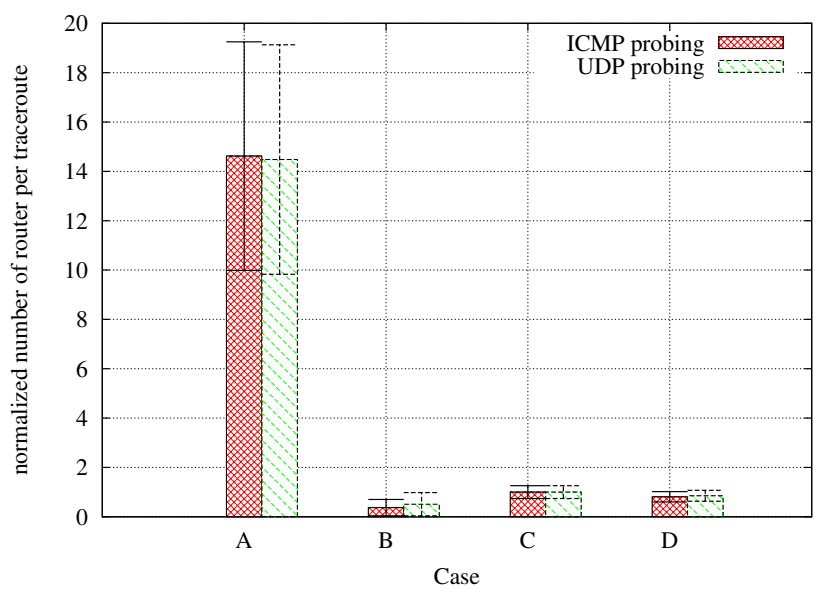

Fig. 7: Normalized (per traceroute) number of routers per case (with standard deviation)

path must remain the same.

Figure $6 \mathrm{~b}$ illustrates two paths being considered as identical by the path matching algorithm when allowing two different consecutive identified routers.

The following section presents large scale measurements gathered from Planet-Lab nodes and a fine-grained analysis of the algorithm results.

\section{Measurement Results}

We first detail the results obtained from 30 Planet-Lab nodes to a set of CAIDA's routed /24 composed of more than 10,000 IP addresses. Then we detail the results obtained from a typical ISP user probing the same set of IP addresses, using ICMP, UDP, but also TCP.

\section{A. Coarse-grain Internet results}

1) Paths characterization: figure 7 represents the normalized number of router per case. On average, a traceroute is composed of 16.78 routers distributed as followed:

- $A$ case - 14.5 routers

- $B$ case -0.45 router

- $C$ case - 1 router

- $D$ case -0.83 router

Figure 8 shows the distribution of cases depending on the distance between the router and the initial source. Note that when handling a "premature end" of a traceroute (i.e. when finishing with five "*”), the $D$ cases are only counted once (it corresponds to the closest router from the source) as explained in section IV-B3.

This figure shows that the closer a router is to the source, $\mathrm{S}$, the mostly it will be of case $A$. On the opposite, the further a router is, the highest the probability to fall in $C$ and $D$ cases, which in turn comes at the expense of a lower probability of the router would be in case $A$. Perhaps not surprisingly, this shows that the longer the path is the higher the chances the routers would behave according to the $D$ unexplicative case. 


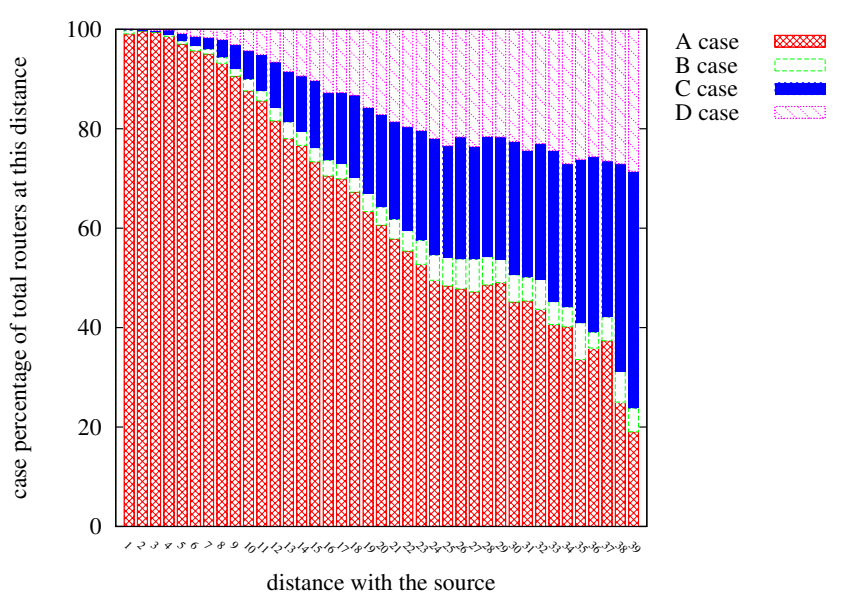

Fig. 8: Case percentage with regards to the distance to the source $S$

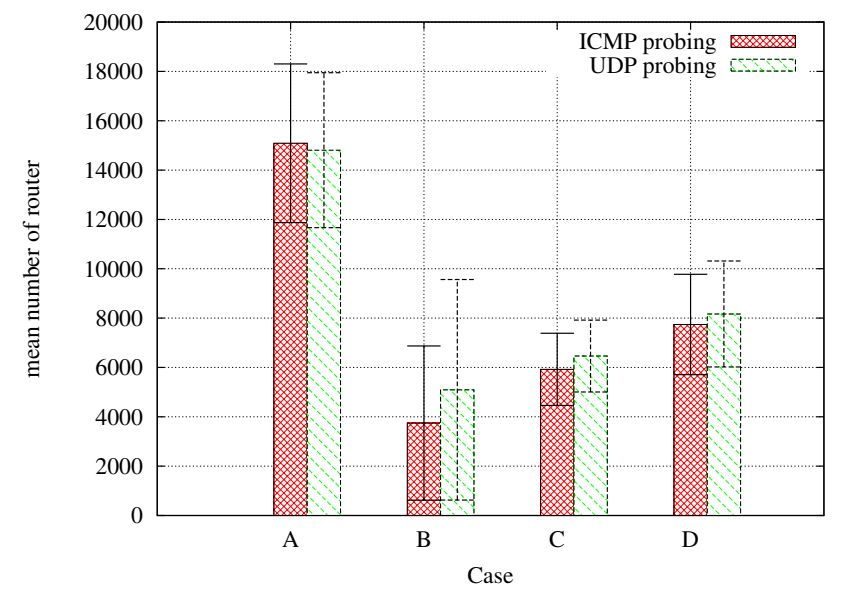

Fig. 9: Mean number of routers per case (with standard deviation)

2) Routers characterization: figure 9 summarizes the classification of routers encountered during the probing of every IP of our destination set, from each Planet-Lab nodes, using the ICMP and UDP protocols. We remind that for the $D$ case, results are divided by 5 due to the "premature end" of a traceroute (section IV-B3). In opposition to the two previous figures, we add that the router seen are counted only once as we want to survey the Internet's average router behavior (previously we were focusing on the path instead).

Figure 10 details the results of the refining algorithm applied to the Planet-Lab outputs. We first configured the path comparison algorithm to perform an exact path matching and then changed it to allow a certain number of different routers along the path. With a strict path matching configuration, we refine $0.16 \%$ (ICMP) and $0.31 \%$ (UDP) of the $B$ case. This ratio grows up to $0.87 \%$ (ICMP) and $1.12 \%$ (UDP) when allowing up to five different consecutive identified routers. This measure (particularly the small difference when allowing five or ten different routers) can be associated to the fact

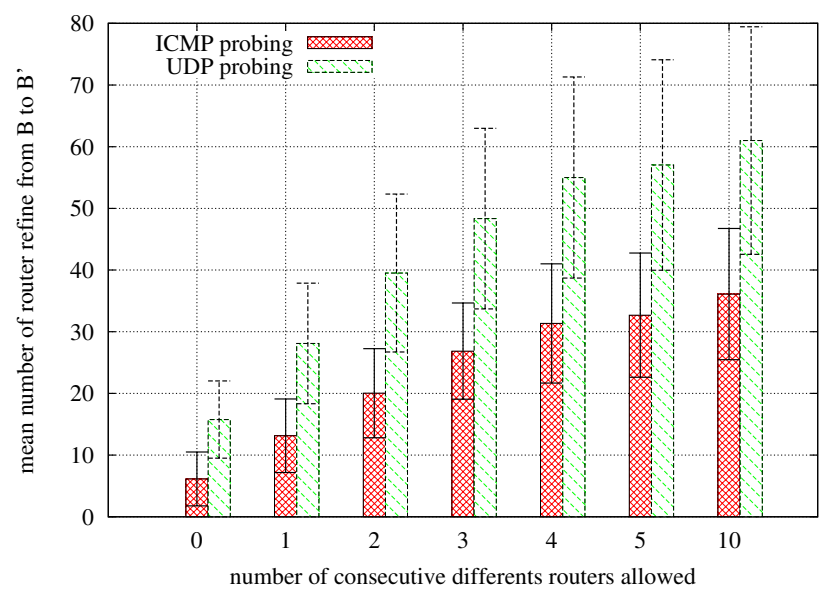

Fig. 10: Mean number of router refined from $B$ to $B^{\prime}$ case depending of the path matching algorithm configuration (with standard deviation)

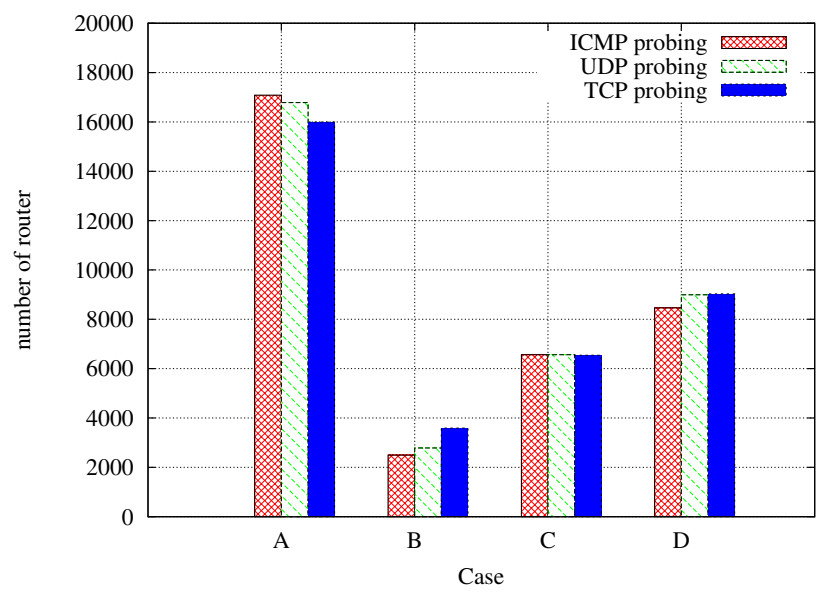

Fig. 11: Number of routers per case with 3 probing protocols

that more than $90 \%$ of MPLS tunnels (which are present in at least $30 \%$ of Internet's traceroute) are at most five hops long[13].

\section{B. Fine-grain results}

Let us now consider the results obtained by a single user using a single host, connected through a given ISP. The set of IP addresses is the same, and we use the ICMP, UDP, but also TCP probing protocols.

Figure 11 presents the results of the classification algorithm applied on the three probing protocolsICMP, UDP and $T C P$. Although the general distribution is globally the same, there are more $A$ cases and fewer $B$ cases than with PlanetLab results (figure 9).

Figure 12 details the refining algorithm used for TCP, which refines TCP results either using ICMP solely, or UDP, or both probing protocols. It illustrates well the fact that, when using multiple probing protocols to refine a single other protocol-based properties output, the results are lower 


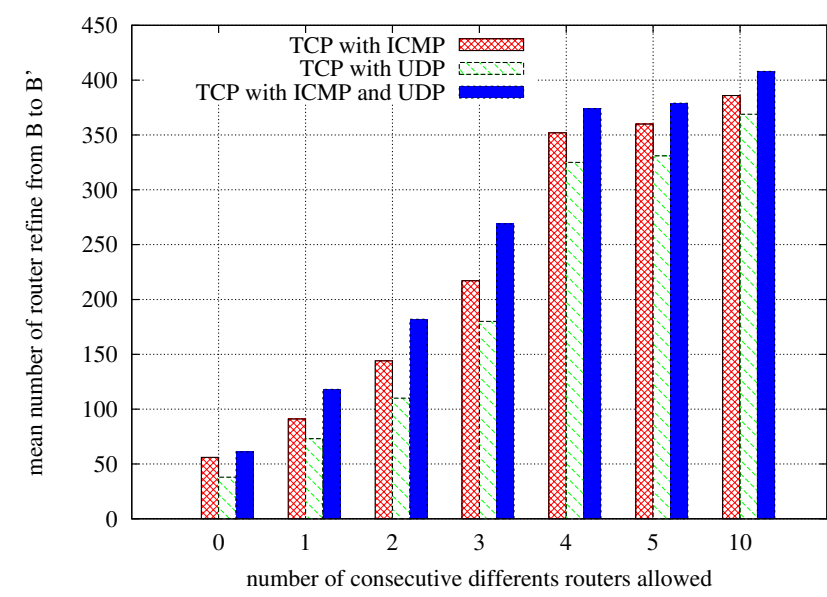

Fig. 12: Refining of TCP probing protocol $B$ case

than what could be expected from the sum of the refining using single protocols outputs, as explained in section III-B3. Indeed, we observe that the improvement of the refining algorithm while using two protocols to refine a single one is small. For instance, when allowing five different consecutive identified routers, the improvement is: $5.2 \%$ when comparing ICMP to ICMP+UDP, and $14.5 \%$ between UDP and ICMP +UDP. We interpret this at a router level by bearing that there is an explicit policy on these routers that forbids it to forge ICMP Time Exceeded packets when the initial protocol is TCP.

Nevertheless, when using both ICMP and UDP to refine the $B$ case of TCP, we see that we are able to completely specify the behavior of $10 \%$ of the routers encountered. Globally, we manage to refine the classification of $1 \%$ of the routers seen.

\section{CONCLUSION}

This work has investigated the problem of ICMP black holes tracking. Our contributions are fourfold: we first introduced a taxonomy for router properties, regarding ICMP. We have shown that three fundamental properties are sufficient to classify Internet routers with respect to the ICMP protocol processing.

Secondly we have introduced a methodology to classify the routers of a path, using a given probing protocol, into four cases. These cases represent the possible logical relationships between the three fundamental properties. Then we introduced a refinement procedure, using two or more probing protocols, that enables to better classify the routers. This approach does not require any control from within the Internet or at destination. It can therefore be immediately ported to any operating system and used by any user.

Third, we designed the IBTrack tool that implements the above algorithm.

Fourth, we performed several large scale experiments to analyze our tool and methodology in real conditions. In these experiments, we also report several insights on the Internet routers ICMP processing.
We therefore believe that this work is a significant step forward. In particular it will help many network administrators to better understand traceroute outputs and debug complex ICMP related problems within routers.

This work deliberately makes very minimalist assumptions on what users might control while processing and diagnosing network failures messages (only the source is controlled). As such, the proposed approach can be easily extended by relaxing some constraints (e.g. the user also controls the destination) or by revisiting some of our assumptions on network functionalities (e.g. the presence of vantage points).

\section{REFERENCES}

[1] "Rfc 1191: Path mtu discovery," http://datatracker.ietf.org/doc/ rfc1191/.

[2] M. Luckie, K. Cho, and B. Owens, "Inferring and debugging path mtu discovery failures," in Proceedings of the 5th ACM SIGCOMM conference on Internet Measurement, ser. IMC '05. Berkeley, CA, USA: USENIX Association, 2005, pp. 17-17. [Online]. Available: http://dl.acm.org/citation.cfm?id=1251086.1251103

[3] M. Luckie and B. Stasiewicz, "Measuring path mtu discovery behaviour," in Proceedings of the 10th annual conference on Internet measurement, ser. IMC '10. New York, NY, USA: ACM, 2010, pp. 102-108. [Online]. Available: http://doi.acm.org/10.1145/1879141. 1879155

[4] N. Egi, M. Dobrescu, J. Du, K. Argyraki, B.-G. Chun, K. Fall, G. Iannaccone, A. Knies, M. Manesh, L. Mathy, and S. Ratnasamy, "Understanding the packet processing capabilities of multi-core servers," 2009. [Online]. Available: http://infoscience.epfl.ch/record/ 134539

[5] M. Dobrescu, N. Egi, K. Argyraki, B.-G. Chun, K. Fall, G. Iannaccone, A. Knies, M. Manesh, and S. Ratnasamy, "Routebricks: exploiting parallelism to scale software routers," in Proceedings of the ACM SIGOPS 22nd symposium on Operating systems principles, ser. SOSP '09. New York, NY, USA: ACM, 2009, pp. 15-28. [Online]. Available: http://doi.acm.org/10.1145/1629575.1629578

[6] "Myricom 10-gigabit ethernet performance measurements," http:// www.myricom.com/scs/performance/Myri10GE/.

[7] E. Katz-Bassett, H. V. Madhyastha, V. K. Adhikari, C. Scott, J. Sherry, P. van Wesep, T. E. Anderson, and A. Krishnamurthy, "Reverse traceroute," in NSDI, 2010, pp. 219-234.

[8] E. Katz-Bassett, H. V. Madhyastha, J. P. John, A. Krishnamurthy, D. Wetherall, and T. Anderson, "Studying black holes in the internet with hubble," in Proceedings of the 5th USENIX Symposium on Networked Systems Design and Implementation, ser. NSDI'08. Berkeley, CA, USA: USENIX Association, 2008, pp. 247-262. [Online]. Available: http://dl.acm.org/citation.cfm?id=1387589.1387607

[9] Y. Hyun, B. Huffaker, D. Andersen, E. Aben, C. Shannon, M. Luckie, and kc claffy, "The caida ipv4 routed /24 topology dataset," http://www.caida.org/data/active/ipv4_routed_24_ topology_dataset.xml, november 2011.

[10] M. J. Luckie, "Scamper: a scalable and extensible packet prober for active measurement of the internet," in Internet Measurement Conference, 2010, pp. 239-245.

[11] B. Augustin, X. Cuvellier, B. Orgogozo, F. Viger, T. Friedman, M. Latapy, C. Magnien, and R. Teixeira, "Avoiding traceroute anomalies with paris traceroute," in Internet Measurement Conference, 2006.

[12] "Planet-lab," http://www.planet-lab.org.

[13] B. Donnet, M. Luckie, P. Mérindol, and J.-J. Pansiot, "Revealing mpls tunnels obscured from traceroute," in SIGCOMM Computer Communication Review, vol. 42, no. 2, Apr. 2012. 